\title{
A Time-Saving Method for Analyzing Permanent Magnet Motors
}

\author{
Sung-Hong Won* ${ }^{*} \mathrm{Ki}-\mathrm{SoO}$ Han $\cdot$ Tae-Heoung Kim
}

\begin{abstract}
This paper presents a unique method for simulating permanent magnet motors without time-consuming numerical methods used in the conventional magnetic circuit method. The conventional method gives us average values like torque and power over specified periods of time, but it is usually very difficult and time-consuming to obtain instantaneous characteristics like cogging torques and torque ripples.

The convolution operations method we present, however, considers relative angle variations of stator magnetic circuits and rotor magnetic circuits. As a result, it makes uses of instantaneous values possible.

The authors compare the new method with the coventional method and verify that calculating cogging torque values and back-emf values is possible with the proposed new convolution method.
\end{abstract}

Key Words : BLDC, FEM, Magnetic Circuit, Convolution, Cogging Torque, Back-Emf

\section{Introduction}

Since permanent magnets were adopted in motors, much research has employed analysis methods of motors. The methods can generally be divided into two categories:

- The first one is the so-called "lumped-sum parameter method" in which the magnetic system is considered as a circuit and average

\footnotetext{
* Main author : the Department of Electrical System, Dongyang Mirae University

Tel : +82-2-2610-5180, Fax : +82-2-2610-1854

E-mail : sagewide@dongyang.ac.kr

Date of submit : 2010. 9. 17

First assessment : 2010. 9. 29

Completion of assessment : 2010. 11. 8
}

magnetic flux values per pole are calculated for obtaining the average torque or power. This method is very convenient and simple to calculate but not easy to get instantaneous torque or back-emf values.

- The other method involves distributed parameter methods like the finite element method (FEM). This method is suitable for analyzing instantaneous values and it is able to consider the magnetic saturation effect of the iron steel core. The analysis of motors using this method is getting popular, but the method takes significantly more times than the lumped-sum parameter method and it needs experienced industry experts[1-2]. 
For improving these methods, some researchers have studied several new methods like the Electro-Magnetic Circuit Network method, but each takes considerable time to obtain results for the same problems[3].

\section{Method Description}

\subsection{Analysis model: HDD Spindle motor}

The authors propose a novel method for analyzing permanent magnet motors using divided magnetic circuits and a convolution method. This new method can calculate permanent magnet motor characteristics like cogging torque and back-emf values which were considered very difficult characteristics to obtain without using some special methods or techniques.

This new method is very powerful because it is able to finish its analysis in less time than the FEM which is laborious and time-consuming

A pole-pair structural period of motor rotor is divided into several parallel magnetic circuits. The stator magnetic circuits of one tooth period including various air-gap permeances - are also composed as parallel circuits. The convolution calculation of these two groups of parallel circuit values can give the motor characteristics of cogging torques or back-emf values.

Fig. 1 shows the HDD spindle motor which uses an outer-rotor type permanent ring magnet and back-yoke outside of the magnet.

Fig.2 shows the inside of the spindle motor. It includes the stator teeth and the ring-type magnet and a back-yoke rotor in the spindle motor. To strengthen the mmf of the magnetic circuit and prevent the leakage flux, the back-yoke is supposed to be outside of the magnet.

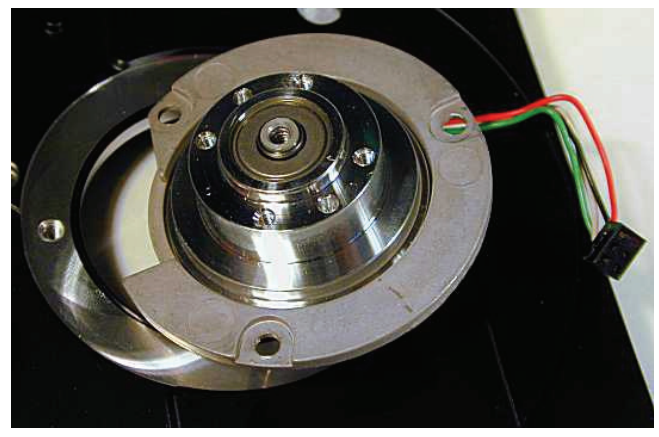

Fig. 1. HDD spindle motor

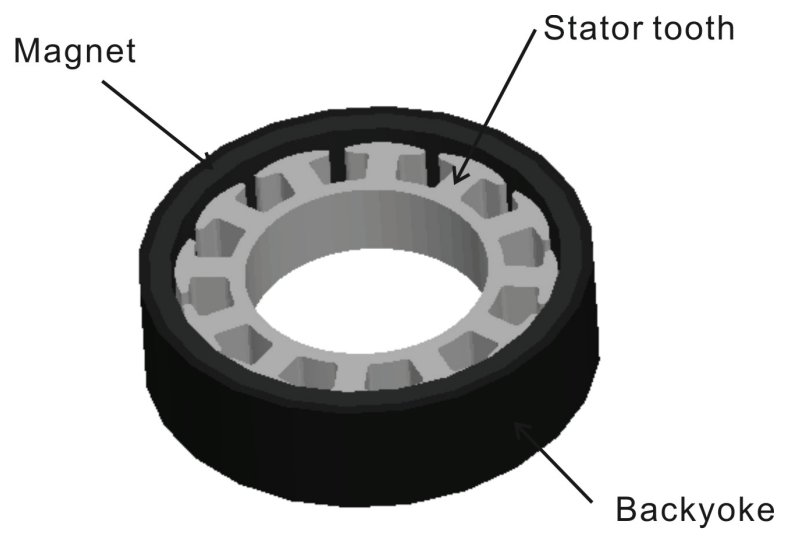

Fig. 2. The stator teeth and the ring-type magnet and back-yoke rotor of the spindle motor

\subsection{Proposed method}

Fig. 3 shows the stator and rotor magnetic circuits of the spindle motor and the concept of the convolution method. Because the magnet is not segmented, there are dead-zones between magnet poles and these dead-zones must be considered when analyzing the motor characteristics.

While the rotor is rotating, the only item that is varying in the magnetic circuits is the airgap permeance. If the discretized magnetic circuit is made like fig. 3 , the reason for the variance of the magnetic circuit permeances is the variance of the airgap length according to the rotation angle.

Journal of KIIEE, Vol.24, No. 11, November 2010 


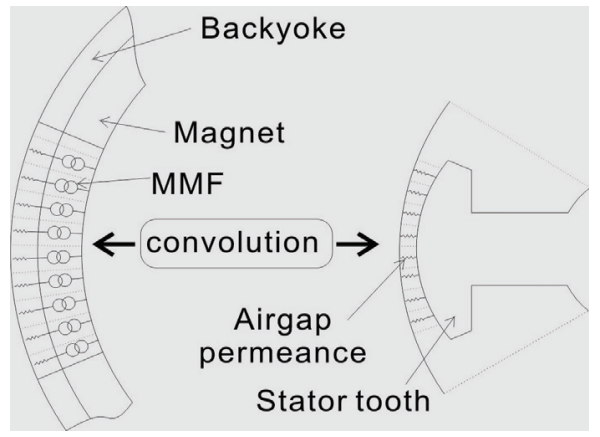

Fig. 3. Stator and rotor magnetic circuits of the spindle motor and the concept of the convolution method

Thus, the convolution sum of the square value of the permanent magnet mmfs and the airgap permeances can make an airgap energy like fig. 4 .

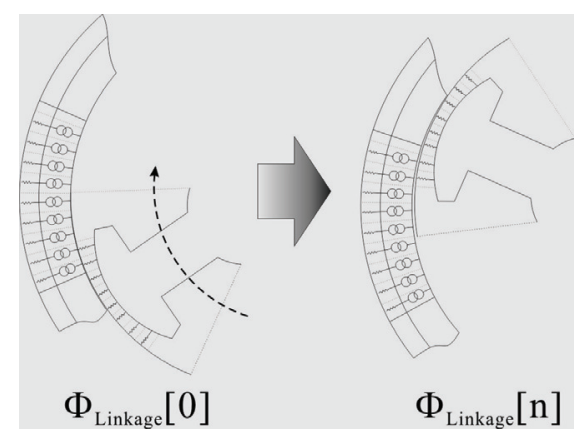

Fig. 4. The convolutions of the stator magnetic circuit and the rotor magnetic circuits

The strongest point in favor of this method is that when the design parameters are changed, the new design factors can be obtained very easily. For example, the cogging torque profile changes can be determined instantaneously when the slot open width (length between a tooth and neighbor tooth) is changed from $1[\mathrm{~mm}]$ to $1.5[\mathrm{~mm}]$. Changes in the magnetization profile and tooth shapes can also be considered by this method instantaneously.

\subsection{Torque \& Back-EMF Calculation}

The following are equations for calculating the airgap energy.

The torque values are easily calculated by the derivation of energy to angle.

$$
\begin{aligned}
& \text { Energy }_{\text {airgap }}[n]=\frac{1}{2} m m f^{2}[n] * \text { Permeance }[n] \\
& \left(n=0 \sim \text { angle }_{\text {polepair }}\right)
\end{aligned}
$$

Permeanc $₫ n]=\mu \frac{S_{a}}{L_{a}}$

$\left\{\begin{array}{l}S_{a}=\text { Area }_{\text {each magneticcircuit }} \\ L_{a}=\text { Length }_{\text {each magnetic circuit }}\end{array}\right.$

$L_{a}=g+R R \times\left[1-\cos \left\{\sin ^{-1}\left(\tan \left(\theta_{r}\right) \times \frac{R R-R s}{R s\left(1-\tan \left(\theta_{r}\right)\right)}\right)\right\}\right]$

g:average airgap length

$R R$ :radius from center to airgap

Rs:radius of tooth

$\theta_{r}$ : angle from tooth center

Torque $=\frac{\Delta \text { Energy }_{\text {airgap }}}{\Delta \theta}$

In equation (1) and (2), $\mathrm{n}$ is the discrete number of the magnetic circuits in a pole pair region.

One of the more difficult aspects of calculating airgap permeances is that actual airgap lengths vary according to the angle $(\Theta r)$ between a magnetic circuit and the center of a stator tooth. They vary in order to reduce the cogging torque and back-emf ripples in the HDD spindle motors.

In this paper, the actual airgap length of each magnetic circuit is derived like equation (3). This airgap length is calculated by a geometrical process deriving the relative length between $\mathrm{RR}$ and $\mathrm{Rs}$ according to the angle Or. The parameters used in equation (3) are shown in fig. 5.

Using a similar process, the back-emf values can be calculated from the airgap flux values. 


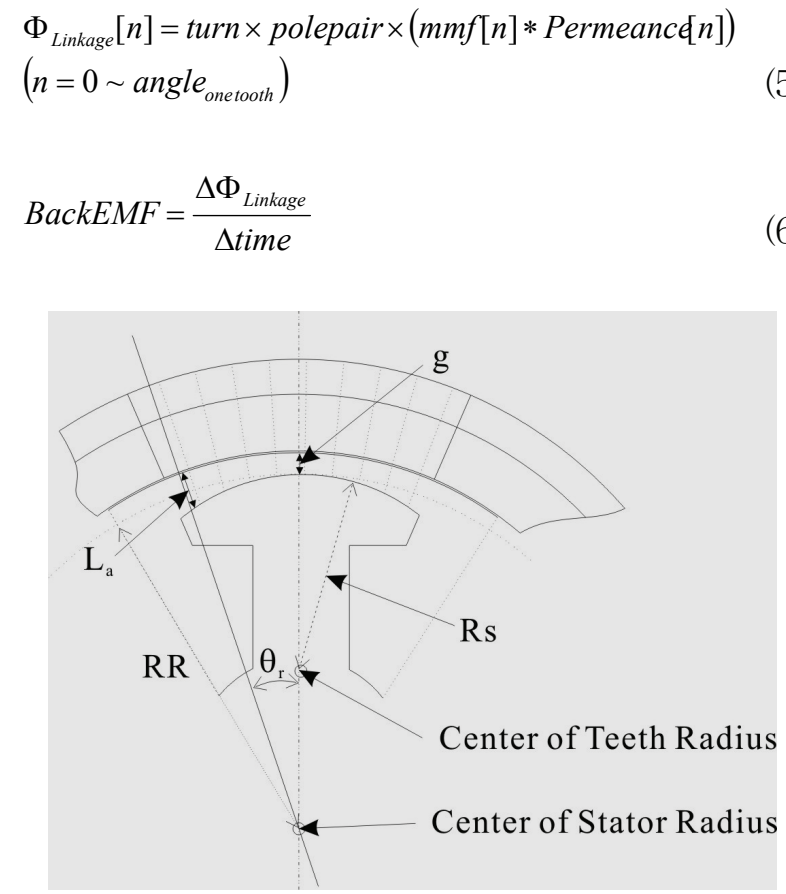

Fig. 5. Parameters used in Equation (3)

\subsection{Comparison of Analysis Results with FEM}

The general method to verify a new method is manufacturing the motor and measuring the cogging torque values and back-emf values and comparing them together. The objective of our proposed method, however, is to present a faster way of calculating instantaneous characteristics of the motor than the FEM. Thus, in this study, a comparison of the proposed method and the FEM is performed.

The cogging torque and the back-emf values of each phase of the motor calculated by the proposed method is compared with the values shown by the FEM analysis. The simulation tool is the Maxwell $\mathrm{EM}$ of Ansoft.

Specifications of the HDD BLDC spindle motor are shown in Table 1.
Table 1. SPECIFICATIONS OF THE MOTOR

\begin{tabular}{|c|c|}
\hline Item & Values \\
\hline Rotor Diameter & $24.3[\mathrm{~mm}]$ \\
\hline Magnet Thickness & $1.35[\mathrm{~mm}]$ \\
\hline Lamination Depth & $5[\mathrm{~mm}]$ \\
\hline Rotation Speed & $5400[\mathrm{rpm}]$ \\
\hline Airgap & $0.25[\mathrm{~mm}]$ \\
\hline Br & $0.8[\mathrm{~T}]$ \\
\hline Turn & 50 \\
\hline
\end{tabular}

Fig. 6. shows the $2 \mathrm{D}$ model of the $\mathrm{HDD}$ spindle motor. In this model, the RR is $12.15[\mathrm{~mm}]$ and Rs is $10[\mathrm{~mm}]$. Thus, the slot open width becomes about $1[\mathrm{~mm}]$.

There are several factors for determining the cogging torque magnitude of a motor. It is not easy to determine the main effect of the cogging torque of a motor. In these types of HDD spindle motors, the magnitude of the cogging torque of the motor is more likely affected by the slot open width than the ratio of $R$ s and $R R$.

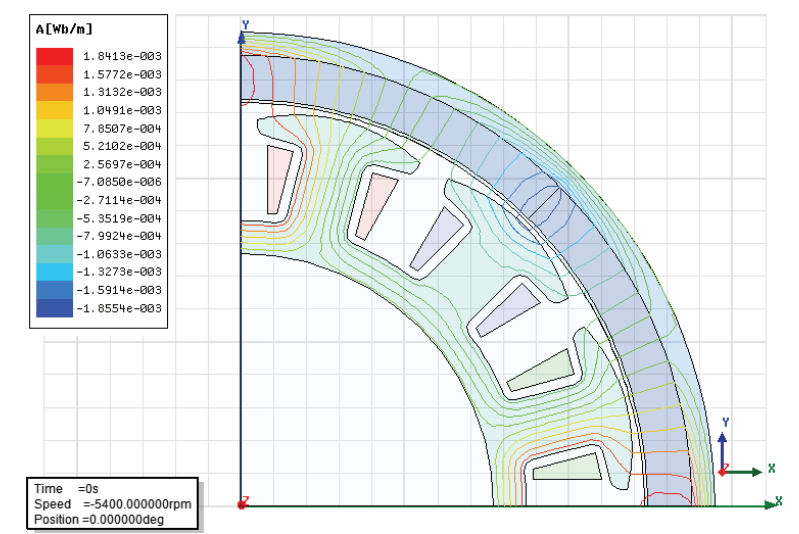

Fig. 6. 2D FEM model and the flux lines

The cogging torque profiles of the proposed method and the FEM are compared in fig. 7. The curve of the proposed method is little higher than the FEM curve, but the shape of the cogging torque profile and the magnitude level are sufficiently 
matched.

One possible reason for this discrepancy is the magnetic saturation of the rotor back-yoke. Generally, the back-yokes are thin due to a reduced rotor inertia. Thus, the back-yokes are always magnetically saturated. However, the magnetic saturation of back-yoke is not considered in the proposed method. Also, the maximum points of the cogging torque profiles are slightly different since the saturated magnetic flux path is not the same as the un-saturated path, but the discrepancy is small.

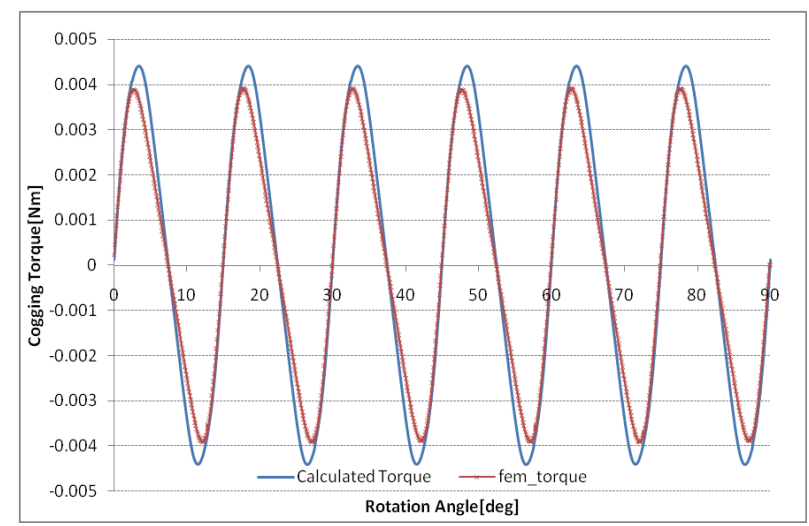

Fig. 7. Comparison of the calculated cogging torque with FEM results

It is very important for engineers to reduce the calculation time for analyzing motor characteristics because, in the initial design process, so many types of motor models need to be calculated to figure out the optimal model for the motor desired. The calculation time of the proposed method is considerably faster than the time required for the FEM analysis. Fig. 8 shows the comparison of the calculation times of two methods.

The FEM analysis with Intel Core2 Duo $3 \mathrm{GHz}$ CPU requires about four minutes but the proposed method can complete calculations in one or less second.

The magnitudes and the shape of the back-emf of each phase are very important characteristics of motors. Fig. 9 shows the back-emf curves calculated by the FEM and fig. 10 shows the curves by the proposed method.

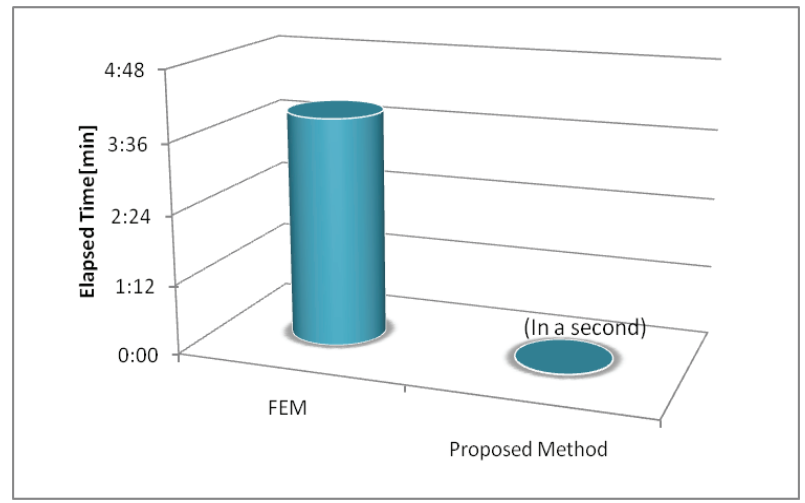

Fig. 8. Comparison of the calculation times

The values by the proposed method are still somewhat bigger than those with the FEM The linkage fluxes by the FEM are reduced because of the back-yoke's magnetic saturation.

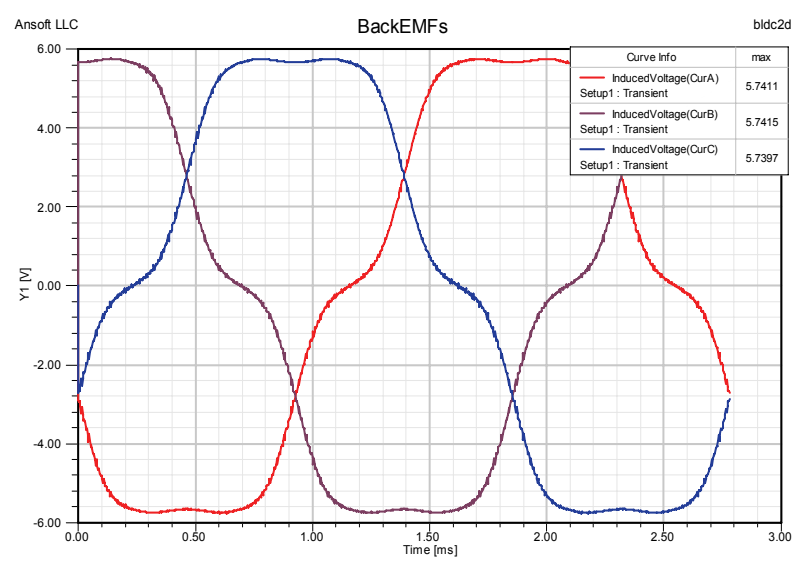

Fig. 9. Back-emf values of each phase with FEM

By adopting an empirical coefficient for the total linkage flux of the magnetic circuit, the calculated results of the proposed method can be matched more to the FEM values. 


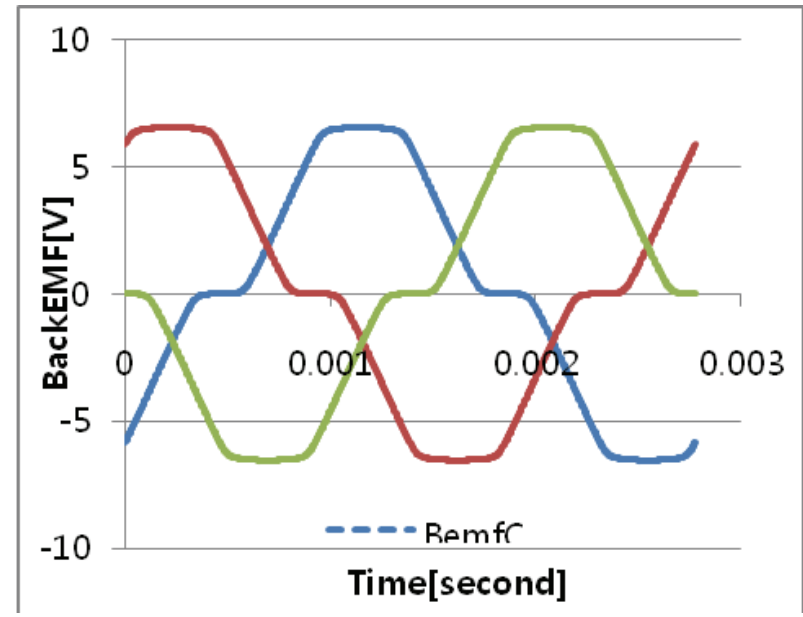

Fig. 10. Calculated back-emf values with the proposed method

\section{Conclusion}

Permanent magnet motors are very important devices in modern industry. Among these motors, the BLDC spindle motors are probably one of the most popular motors in the world. It is therefore important to find new and faster methods of predicting the performance of BLDC motors than the time required for the FEM analysis.

In this paper, a new method for analyzing instantaneous characteristics of BLDC motors with simple convolution sums of magnet mmfs and airgap permeances is compared with the FEM calculation method. The new method gave reliable results immediately.

This paper is particularly useful to engineers who want to know motor characteristics quickly and to know how the characteristics vary according to the geometrical changes of the motor cores.

\section{Acknowledgement}

This paper is financially supported by Dongyang Mirae University Research Grant.

\section{References}

[1] Seung-Chan Park, Byung-Il Kwon, Hee-Soo Yoon, Sung-Hong Won, Young-Gyu Kang, "Analysis of Exterior-rotor BLDC Motor Considering the Eddy Current Effect in the Rotor Steel Shell”, IEII Trans. Magn , vol. 35, no. 3, pp.1302-1305, May. 1999.

[2] Seung-Chan Park, Tae-Ho Yoon, Byung-II Kwon, Hee-Soo Yoon, Sung-Hong Won, "Influence on Brushless DC Motor Performance Due to Unsymmetric Magnetization Distribution in Permanent Magnet", Trans.on Magentics, vol.36, no.4, pp.1898-1901, Jun. 2000.

[3] Yon Do Chun, Jae-Eung Oh, Yasushi Fujishima ,Shinji Wakao, Yun-Hyun Cho, Ju Lee, "Comparison between three-dimensional .3D. equivalent magnetic circuit network method and 3D finite element method for magnetic-field computation”, Journal of Applied Physics, vol. 97, no. 10, pp.10E105-10E105-3, May, 2005.

\section{Biography}

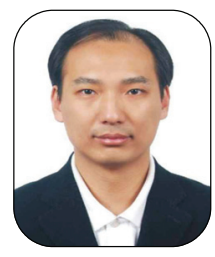

\section{Sung-Hong Won}

He received his B.S., M.S. and Ph. D degree in electrical engineering from Hanyang University in 1993, 1995 and 2007, respectively. He was a senior researcher in Central R\&D center of Samsung electro-mechanics from 1995 to 2004. He is currently an Full-time Lecturer in Dept. of Electrical System at Dongyang Mirae University, Seoul, Korea

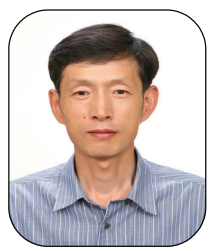

\section{Ki Soo Han}

He received his B.S., M.S. degree in electrical engineering from Seoul National University in 1982 and 1984, respectively. He was a senior researcher in $R \& D$ center of $L G$ electronics from 1984 to 1991, and Daeshin information \& communication research center from 1991 to 1999. Since 1999, He has been an Associate Professor in Dept. of Electrical System at Dongyang Mirae University, Seoul, Korea

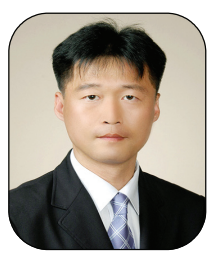

\section{Tae Heoung Kim}

He received his B.S., M.S., and Ph. D. degrees in Electrical Engineering from Hanyang University, Korea, in 1993, 1995, and 2005, respectively. From Jannuary 1995 to August 2002, he worked for LG Electronics as a senior research engineer. Since 2005, he has been a professor in the department of electrical engineering. His research interest is the design of electric machines. 